\title{
ROLE OF COMPUTED TOMOGRAPHY IN CERBROVASCULAR ACCIDENT
}

\author{
V. B. Kalra, P.S.S. Kiran
}

1. Professor, Department of Radio Diagnosis, Konaseema Institute of Medical Sciences, Amalapuram.

2. Senior Resident, Department of Radio Diagnosis, Konaseema Institute of Medical Sciences, Amalapuram.

\section{CORRESPONDING AUTHOR}

Dr. P.S.S. Kiran,

KIMS Medical College,

Amalapuram - 533201.

E-mail: drpsskiran@gmail.com

Ph: 00919490119999

ABSTRACT: Cerebrovascular accident is characterized by the sudden loss of blood circulation to an area of the brain resulting in a corresponding loss of neurologic function. Stroke is mainly of two types ischaemic or haemorrhagic and differentiation is important as the management differs substantially with the type of stroke. Aim of the study was to compare the clinical diagnosis with computed tomography diagnosis and to differentiate between the types of stroke.

The study included 90 clinically suspected patients of stroke who underwent CT imaging at Konaseema Institute of Medical Sciences, Amalapuram, A.P. from July,2009 to September,2011.The incidence of CVA was significantly higher in males than females $73.3 \%$ $\mathrm{n}=66$ vs $26.6 \% \mathrm{n}=24)$. The most frequently affected age group was 50 to 69Years of age(67\%)The incidence of ischaemic stroke was significantly higher than haemorrhagic stroke both in males and females.

The most common localizations were parietal and frontal lobe. The middle cerebral artery followed by the anterior cerebral artery were the commonest vascular territories affected.

The sensitivity of clinical diagnosis remained $90 \%$.But clinical diagnosis is not dependable for therapeutic \& prognostic purpose when planning for anti-coagulation or thrombotic therapy.

INTRODUCTION: Cerebrovascular accident is a major cause of morbidity and mortility. Clinically it is difficult to differentiate the types of stroke (Haemorrhagic or ischemic) in majority of cases as there are no specific differentiating features.

CVA is most severe, sudden in onset and requires emergency imaging for specific diagnosis. Haemorrhagic stroke is due to rupture of vessels, aneurysm in majority cases associated with hypertension. Whereas the underlying cause in ischaemic stroke is thrombotic or embolic occlusion of a intracranial vessel. To differentiate the types of stroke is very vital as the management and functional outcome depends upon the specific diagnosis.

MATERIALS AND METHODS-The study was carried out in Konaseema Institute of Medical sciences from July 2009 to September 2011.

After detailed clinical history \& physical examination the patients were scanned in the radiology department using GE spiral CT. From base of skull to sella $2 \mathrm{~mm}$ sections obtained and above sella $5 \mathrm{~mm}$ sections were taken. The data were analysed statistically and clinical features and computed tomography findings were correlated in patients with cerebrovascular accident. 
RESULTS-A total of 90 patients with clinical presentation of stroke were subjected for CT scan ,out of which 80 had positive findings and rest 10 had normal CT. Brain tumor and cerebral aneurysm was observed in one each.

The age range was from 30 to 85 years with 66(73.3\%) males and $44(26.6 \%)$ females. Male to female ratio of $2.75: 1$ as shown in table 1 .Majority of patients were above 50 years of age.

Ischaemic stroke was most common in both males and females constituting total $56(62.2 \%)$ and haemorrhagic CVA 21(26.6\%)

DISCUSSION- Cerebrovascular disease is the third common cause of morbidity \& mortality after cancer and cardiac disease in elderly patients. Incidence of CVA increases with age with a male predominance, which was similar to the most of the studies reported in literature (warlow et al) With the advancement of age the incidence of atherosclerosis and diseases like hypertension, diabetes mellitus increases that would explain the higher incidence of stroke in elderly patients.

We found that most risk factor in haemorrhagic stroke was hypertension $60 \%$ (Ali 56\%, Khawaja 56.8\%).Presence of diabetes mellitus was confirmed in $28 \%$ of ischaemic patients.

The haemorrhagic stroke is sudden and presents with symptoms like headache, vomitting and deterioration of conscious level. On the other hand ischaemic stroke has gradual onset and symptoms progress over a period of time. CT sensitivity is $100 \%$ in haemorrhage and other lesions which can mimic like CVA. Acute haemorrhage appears as hyperdense on CT scan. Most common localization in both types of stroke was parietal lobe followed by frontal lobe.

Sensitivity of ischaemic stroke is around $80 \%$ and still less in first 24 hours. Few patients with early ischaemic stroke showed signs like loss of grey white differentiation, hyperdense MCA \& insular ribbon sign. However to appreciate the early signs of ischaemic stroke is not always easy and requires a great experience.

MRI with diffusion-weighted imaging in ischaemic stroke is more reliable than CT. However still CT remains the first choice of investigation in cases of stroke because of inexpensive technique, speedy \& wide availability in all hospitals.

Moreover there are no contraindications of CT scan like presence of pacemaker, ventilator \& mechanical implants.

\section{REFERENCES:}

1. WarlowC. Cerebral vascular disease.Med International 1987;47:1919-27

2. Von Kumar et al .Acute stroke.Radiology 1999;212,307-324

3. Ali L.Jamil H.,Mashroor AS.Factors in stroke.J.Coll.Physicians Surg.Pakistan

4. An auditing of brain computed tomograms of CVA patientsUduma,F.U.Okere,P.C.N.,Motah,M..Journal of Medicine and Medical Sciences Vol 2(9)PP 1075-1079 Sept,2011

5. Provenzole J.P. et al.Assessment of the patients with hyperacute stroke,image \& therapy:Radiology 2003;229:347-359

6. Harrison MJG,Clinical distinction of cerebral haemorrhage and cerebral infarction.Postgraduate Med.J.1980,56: 629-32

7. Jehangir Khan,Atique UR Rehman. Comparision of clinical diagnosis with computed tomography in ascertaining type of stroke J.Ayub Med.Coll.Abbottabad,2005;17(3) 


\section{ORIGINAL ARTICLE}

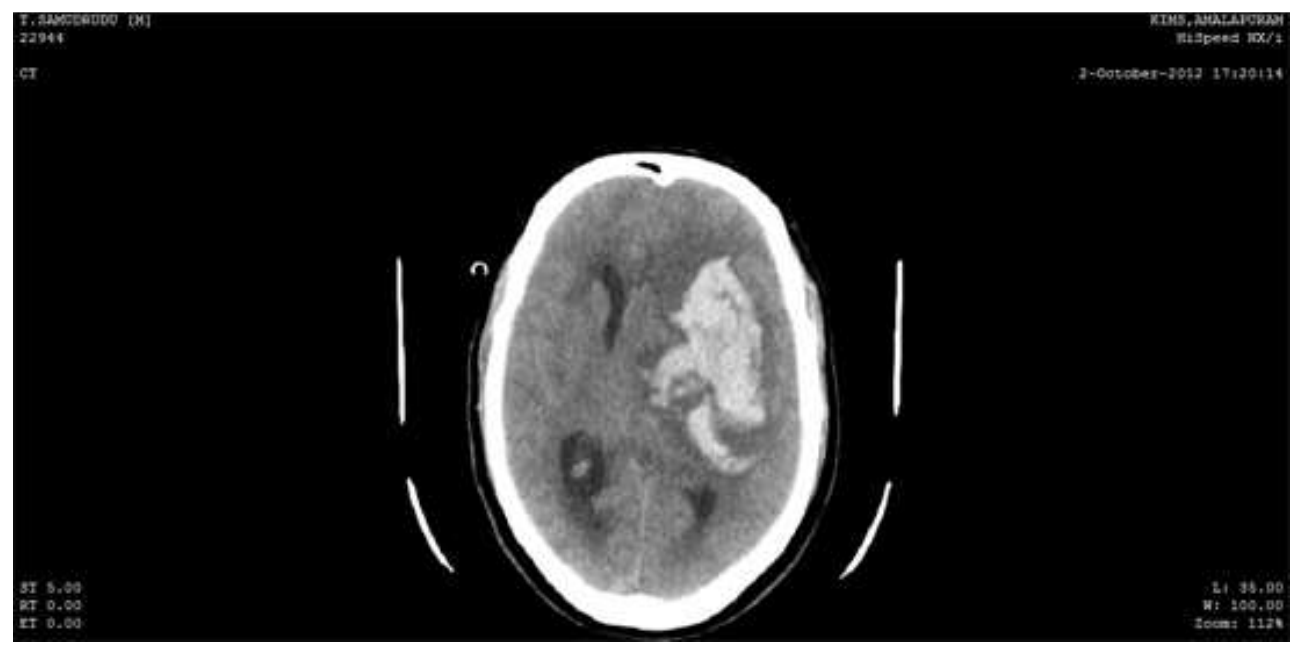

Haemor

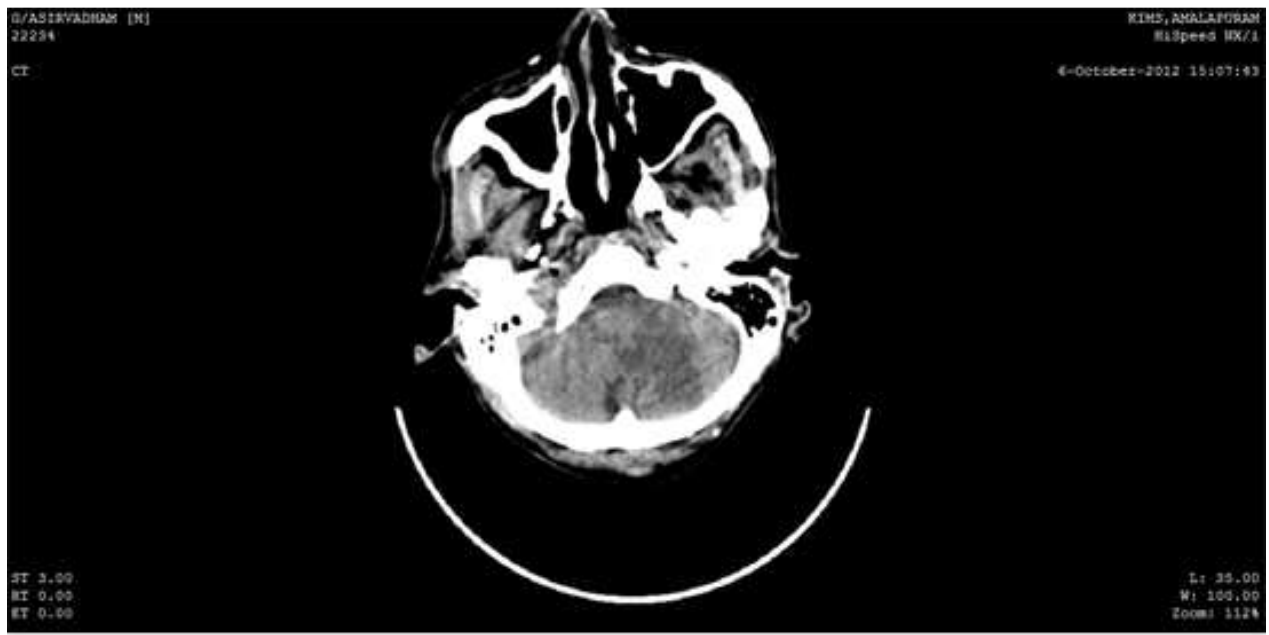

left-cerebellar-infarct

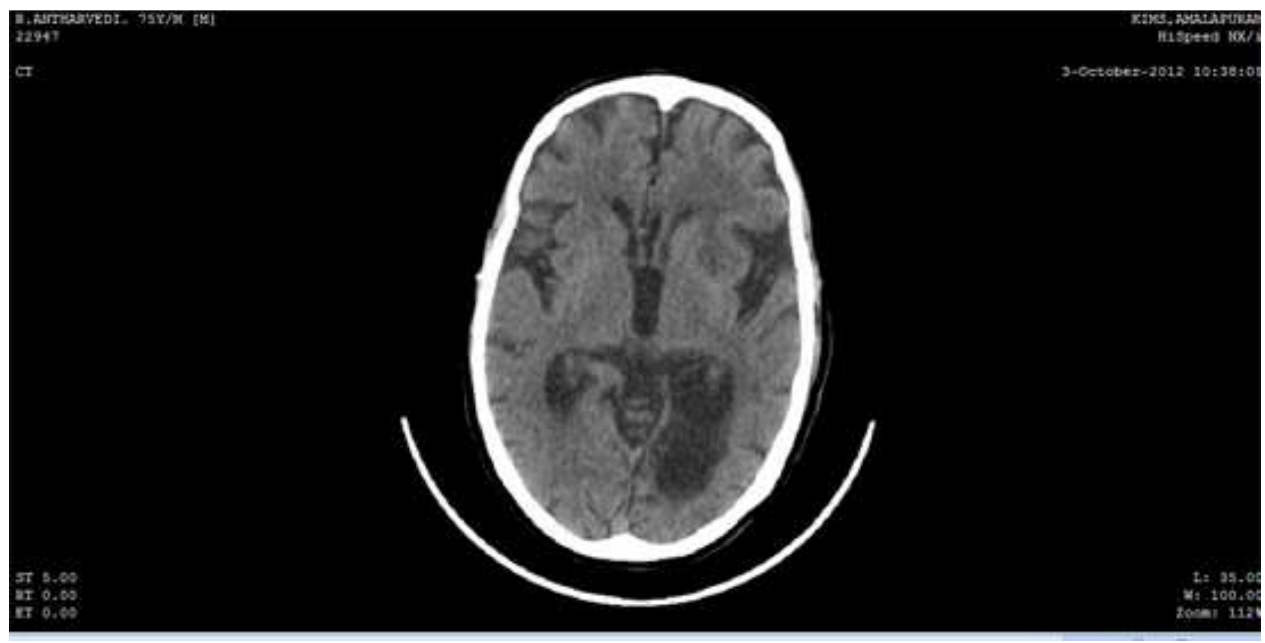

left occipital lobe infarct 


\section{ORIGINAL ARTICLE}

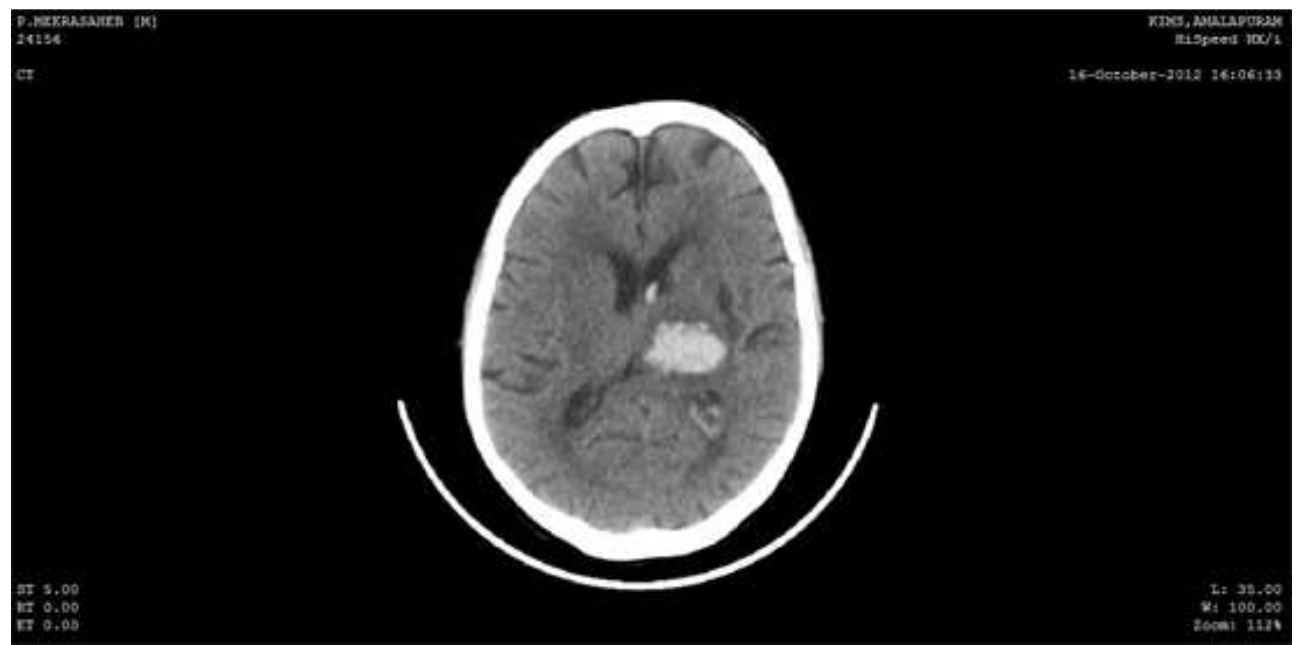

left thalamic bleed

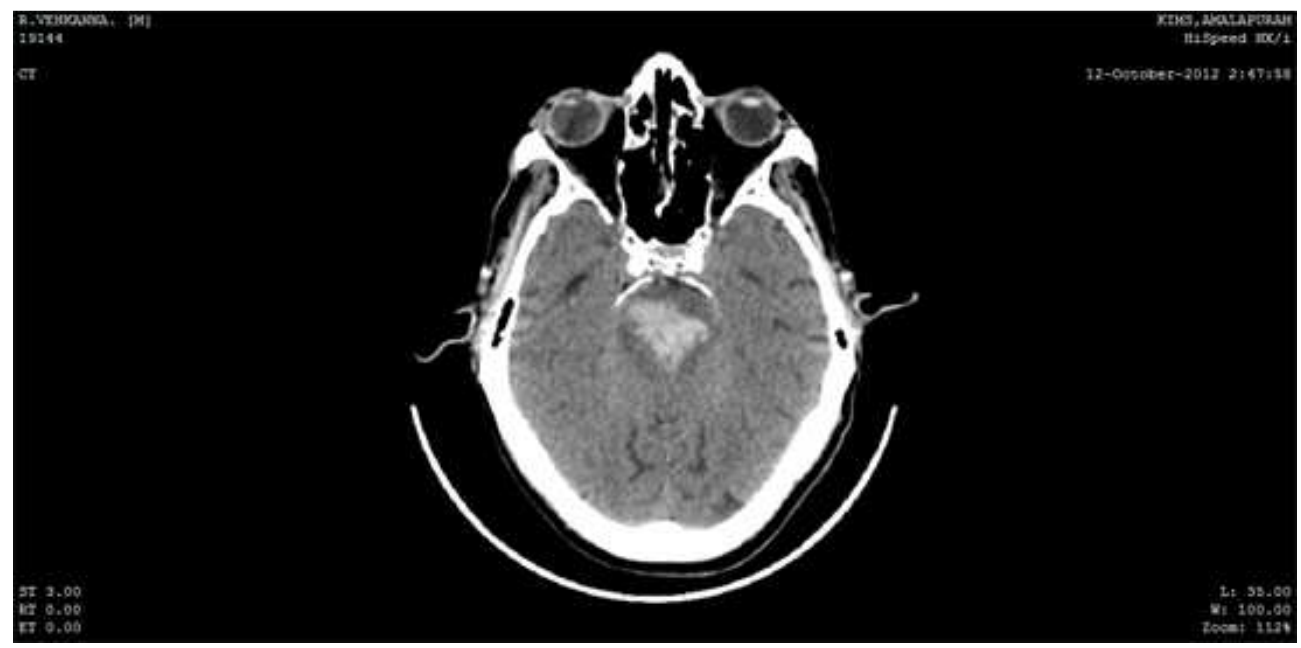

pontine bleed

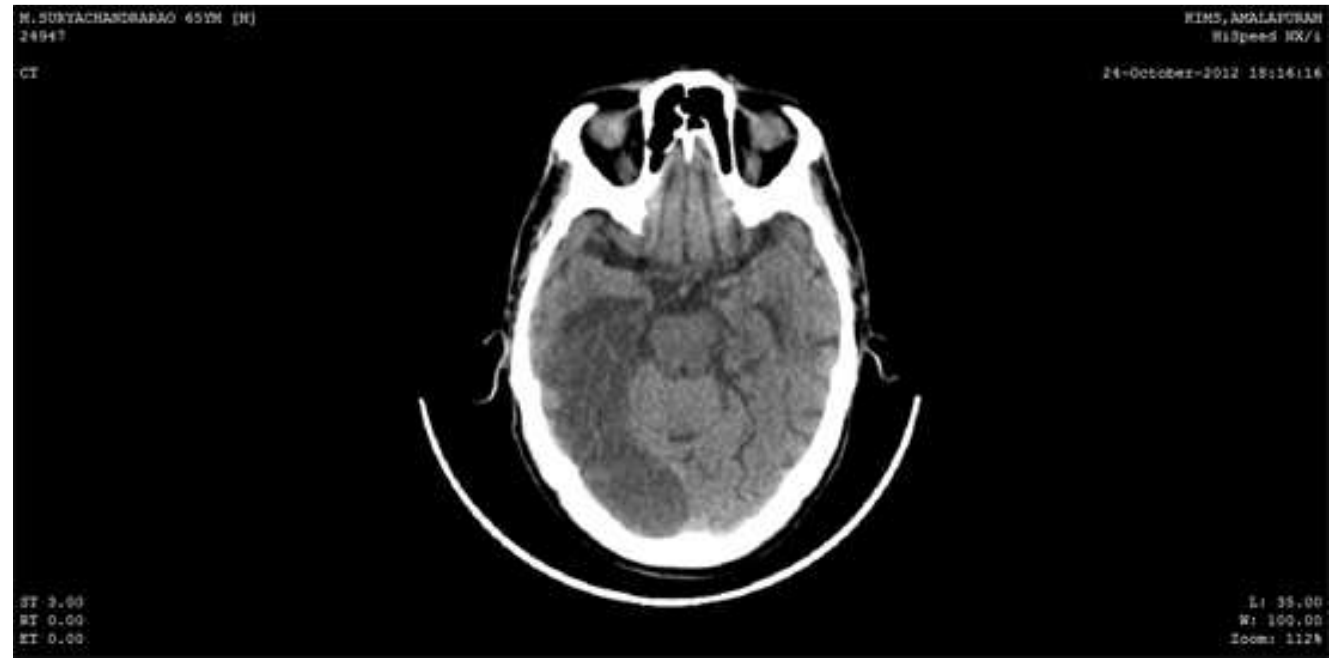

right pca territory infarct 


\section{ORIGINAL ARTICLE}

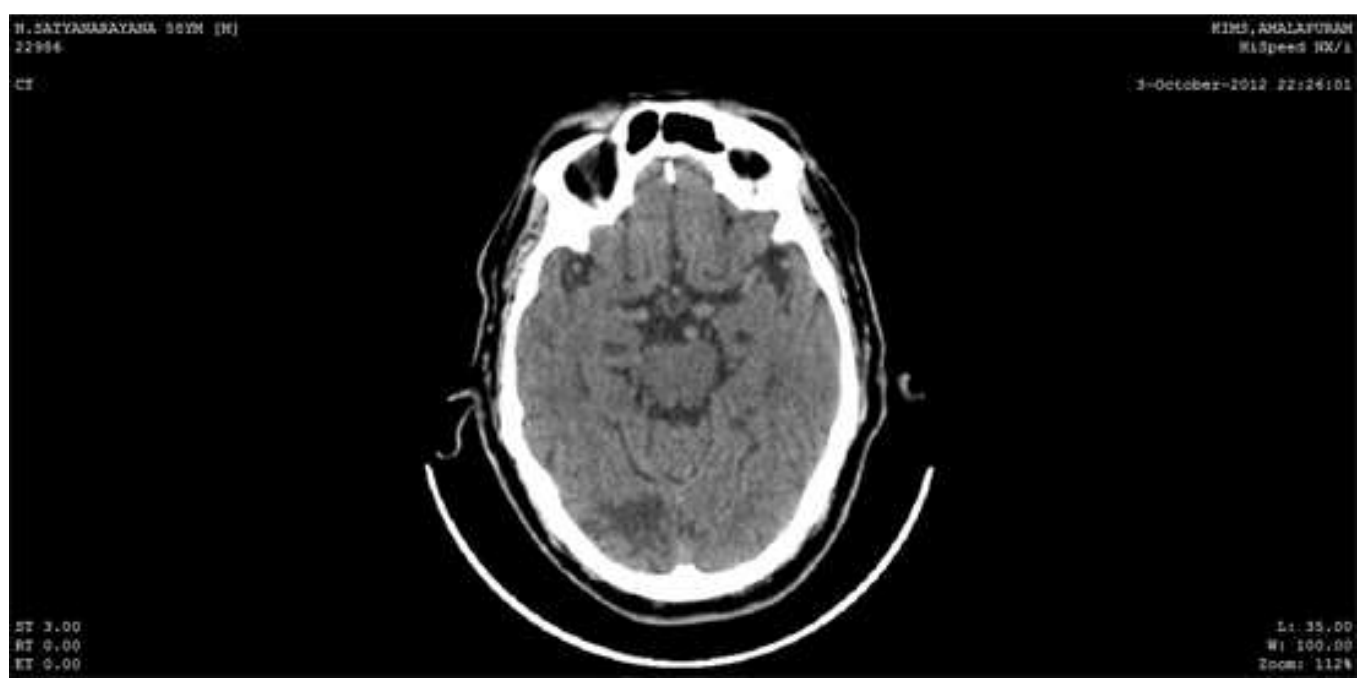

right temporal lobe infarct 